\title{
Descriptive analysis of sepsis in a developing country
}

\author{
Gilbert Abou Dagher, Mothana Saadeldine, Rana Bachir, Dina Zebian and Ralphe Bou Chebl
}

\begin{abstract}
Background: Most studies on sepsis were conducted in developed countries. The aim of this study is to report on a series of patients with sepsis in a tertiary hospital in a developing country.

Methods: Patients admitted through the emergency department of a single university-based institution between January 2008 and June 2012, with a final diagnosis of sepsis, bacteremia, or septic shock, were retrieved. A sample of 97 patients was selected. Vital signs at presentation, number of SIRS criteria, use of vasopressors and steroids, and in-hospital mortality were recorded.

Results: The mean age was $70.09 \pm 16.82$, ranging from 19 to 96 years; $48.5 \%$ were females and $51.5 \%$ were males; $42.3 \%$ of the patients were found to be bacteremic. IV fluid requirement during the first $6 \mathrm{~h}$ was $1.75 \pm 1.96 \mathrm{I}$. The time for antibiotic initiation was $3.43 \pm 4.48 \mathrm{~h}$, with $87.6 \%$ of the antibiotics initiated in the emergency department. Norepinephrine was the most commonly used vasopressor (38.1\%) followed by dopamine (8.2\%), and the inotrope dobutamine (4.1\%); $45.3 \%$ of the patients were admitted to the intensive care unit (ICU), and the remaining $54.7 \%$ were managed on the general practice unit (GPU). A total of 30 (30.9\%) septic patients died. The 28-day mortality was $20.6 \%$. Deceased patients had greater vasopressor use, a longer stay in the ICU $(p=0.001)$, and a longer time to norepinephrine use $(p=0.004)$.

Conclusions: This is the first study providing an in-depth analysis of sepsis patients in a developing country, looking at in-hospital mortality, SIRS criteria utility, and at the overall sepsis management.
\end{abstract}

\section{Background}

Sepsis has gained worldwide recognition in the last two decades. The Early Goal-Directed Therapy (EGDT) study introduced protocol-based care in sepsis management in 2001 [1], followed by the Surviving Sepsis Campaign (SSC) in 2002 [2], to the NIH-funded PROCESS and ARISE trials that looked at the value of such protocol-based intervention [3, 4]. Despite the enormous amount of sepsis research, the incidence of affected patients in the USA has doubled in the past decade, and approximately 750,000 persons are affected annually [5], with one septic patient presenting to an emergency department in the USA every minute [6]. Most sepsis studies, whether evaluating the overall mortality of the disease or the benefit of a specific treatment modality, were done in developed countries such as the USA,

\footnotetext{
* Correspondence: rb94@aub.edu.lb

* Correspondence: rb94@aub.edu.lb Lebanon
}

Australia, and Europe. There are no studies looking at sepsis, the value of SIRS criteria, or its related mortality in a developing country. The aim of this study is to describe the pathogens, underlying medical conditions, management, and mortality of patients presenting with sepsis at the American University of Beirut Medical Center (AUBMC), a 420-bed teaching hospital in Lebanon.

\section{Methods}

A separate study at our institution looked at sepsis in dialysis patients and looked at their in-hospital mortality. There were a total of 97 end-stage renal disease (ESRD) patients. The non-ESRD patient control group was significantly larger. A convenience sample of 97 patients admitted through the Emergency Department of a single university-based institution between January 2008 and June 2012, with a final diagnosis of sepsis, bacteremia, or septic shock, was randomly selected with the help of a computer-generated program. Exclusion criteria included age less than 18 years, pregnant, and trauma cases. After 
an Institutional Review Board (IRB) approval from the American University of Beirut, we studied that same cohort of patients and looked at a variety of parameters related to their emergency department (ED) management, disposition, and outcome.

Age, gender, ethnicity, and history of comorbidities were obtained from the patients' medical record. Patients' vital signs and number of SIRS criteria were collected at initial presentation to the emergency department. The site of infection, causative microorganism, and presence of bacteremia were retrieved as well as complete blood count, electrolytes, lactate, cardiac enzymes, arterial blood gas results, and coagulation profile results. Time to antibiotics and amount of fluid resuscitation within the first 6 and $24 \mathrm{~h}$, duration and type of vasopressors, and steroids administration were also noted. Disposition from the ED, length of stay in ED, intensive care unit (ICU), or general floor, was calculated. We also looked at hospital mortality as well as 28-day mortality.

\section{Statistical analysis}

A two-tailed sample $t$ test compared lengths of stay in ED, ICU, or floor; overall length of stay in hospital; time to and duration of vasopressors, antibiotics, and steroids; fluid replacement at 6 and $24 \mathrm{~h}$, and vital signs at presentation and after $6 \mathrm{~h}$, between deceased patients and non-deceased. A Pearson's chi-square test was used to compare differences in distribution of bacteremia, ED disposition, use of vasopressors or steroids, and number of SIRS criteria at presentation between deceased patients and non-deceased. Statistical analyses were performed using SPSS Statistics for Windows Version 21.0. (Armonk, NY: IBM Corp).

\section{Results}

\section{Patient characteristics}

The mean age was $70.09 \pm 16.82$, ranging from 19 to 96 years; $48.5 \%$ were females and $51.5 \%$ were males. The most common underlying comorbidity was hypertension $(58.8 \%)$ followed by diabetes $(34.0 \%)$ and coronary artery disease $(25.8 \%)$ (Table 1$)$. There was no significant difference in comorbidity distribution between patients presenting with a systolic blood pressure less or more than $90 \mathrm{mmHg}$. Systolic blood pressure upon presentation to the emergency department ranged from 53 to $180 \mathrm{mmHg}$, while it ranged from 66 to $177 \mathrm{mmHg}$ after $6 \mathrm{~h}$ of presentation.

\section{Microbiology}

Bacteremia was defined as a single positive blood culture showing non-skin flora pathogens or a minimum of two positive blood culture bottles with skin flora pathogens; $42.3 \%$ of the patients were found to be bacteremic. The most common sites of infection were genitourinary
Table 1 Baseline characteristics of the patients

\begin{tabular}{|c|c|}
\hline Age (years) & $70.09 \pm 16.8$ \\
\hline \multicolumn{2}{|l|}{ Sex (\%) } \\
\hline Female & 48.5 \\
\hline Male & 51.5 \\
\hline \multicolumn{2}{|l|}{ Chronic coexisting conditions (\%) } \\
\hline Hypertension & 58.8 \\
\hline Diabetes & 34 \\
\hline Coronary artery disease & 25.8 \\
\hline Congestive heart failure & 16.9 \\
\hline Chronic obstructive lung disease or emphysema & 10.3 \\
\hline Neurologic disease & 3.1 \\
\hline \multicolumn{2}{|l|}{ SIRS criteria at presentation (\%) } \\
\hline 2 or more & 67 \\
\hline Less than 2 & 30 \\
\hline \multicolumn{2}{|l|}{ Vital signs upon presentation } \\
\hline Temperature $\left({ }^{\circ} \mathrm{C}\right)$ & $37.55 \pm 1.127$ \\
\hline Systolic blood pressure $(\mathrm{mmHg})$ & $111.23 \pm 27.63$ \\
\hline Diastolic blood pressure (mmHg) & $60.81 \pm 16.91$ \\
\hline Mean arterial pressure $(\mathrm{mmHg})$ & $77.27 \pm 18.96$ \\
\hline Heart rate (beats/min) & $100.43 \pm 28.67$ \\
\hline Respiratory rate (respiration/min) & $22.31 \pm 6.25$ \\
\hline Oxygen saturation (\%) & $94.07 \pm 6.7$ \\
\hline \multicolumn{2}{|l|}{ Baseline laboratory values } \\
\hline White cell count (per $\mathrm{mm}^{3}$ ) & $19.5 \pm 3.34 \times 10,000$ \\
\hline Hemoglobin & $10.69 \pm 2.45$ \\
\hline Hematocrit & $32.27 \pm 8.43$ \\
\hline Lactate (mmol/l) & $3.80 \pm 3.531$ \\
\hline Creatinine (mg/dl) & $1.86 \pm 1.9$ \\
\hline Blood urea nitrogen (mg/dl) & $37.03 \pm 26.29$ \\
\hline Total bilirubin (mg/dl) & $3.08 \pm 5.2$ \\
\hline Partial pressure of carbon dioxide $(\mathrm{mmHg})$ & $36.42 \pm 10.8$ \\
\hline pH arterial blood & $7.36 \pm 0.13$ \\
\hline $\mathrm{PaO}_{2} / \mathrm{FiO}_{2}$ & $241.8 \pm 111.54$ \\
\hline \multicolumn{2}{|l|}{ Bacteremia } \\
\hline Yes & 41 \\
\hline No & 56 \\
\hline \multicolumn{2}{|l|}{ Site of infection (\%) } \\
\hline Urine & 40.2 \\
\hline Lung & 19.6 \\
\hline Unknown & 19.6 \\
\hline Skin & 10.3 \\
\hline $\mathrm{Gl}$ & 8.2 \\
\hline Endocarditis & 1 \\
\hline Oral flora & 1 \\
\hline
\end{tabular}


Table 2 Causative microorganisms

\begin{tabular}{lc}
\hline Microbiology & $N(\%)^{\mathrm{a}}$ \\
\hline Escherichia coli & $37(38.1)$ \\
Klebsiella pneumoniae & $11(11.3)$ \\
Coagulase-negative staphylococci & $8(8.2)$ \\
Pseudomonas aeruginosa & $7(7.2)$ \\
Enterococcus species & $6(6.2)$ \\
Candida species & $6(6.2)$ \\
Non-albicans Candida & $4(4.1)$ \\
Proteus mirabilis & $3(3.1)$ \\
Diphtheroids species & $3(3.1)$ \\
Acinetobacter baumannii & $2(2.1)$ \\
Streptococcus pneumoniae & $2(2.1)$ \\
Others Clostridium species, Listeria monocytogenes, & $11(11.3)$ \\
Morganella morganii, Brucella species, & \\
Providencia alcaligenes &
\end{tabular}

${ }^{a}$ More than one organism may be retrieved from a single subject

(40.2 \%) followed by pulmonary (19.6\%) and integumentary $(10.3 \%)$. In $19.6 \%$ of cases, no focus of infection could be identified (Table 1). In 76 out of 97 patients (78.4\%), a microorganism was identified with Gram-negative organisms exceeding the Gram-positives. Among the Gram-negative organisms, Escherichia coli has been consistently the most commonly isolated organism (38.1 \%) followed by Klebsiella (11.3 \%) and Pseudomonas (7.2\%). In the Gram-positive bacteremia group, coagulasenegative staphylococci represented the majority of the isolates $(8.2 \%)$ (Table 2). When stratified according to blood pressure, $38.5 \%$ of patients with systolic blood pressure less than $90 \mathrm{mmHg}$ were bacteremic in contrast to $43.5 \%$ of patients with a systolic blood pressure greater than $90 \mathrm{mmHg}$.

\section{Management}

IV fluid requirement during the first $6 \mathrm{~h}$ was $1.75 \pm$ $1.96 \mathrm{l}$ and $3.37 \pm 2.85$ during the first $24 \mathrm{~h}$. On the other hand, the time for antibiotic initiation was $3.43 \pm 4.48 \mathrm{~h}$, with $87.6 \%$ of the antibiotics initiated in the emergency department (Table 3).

The time to initiate vasopressors was $9.14 \pm 8.03 \mathrm{~h}$. Norepinephrine was the most commonly used vasopressor $(38.1 \%)$ followed by dopamine $(8.2 \%)$ and the inotrope dobutamine $(4.1 \%)$. Vasopressor treatment duration in the first $24 \mathrm{~h}$ was $3.33 \pm 3.411 \mathrm{~h}$. Furthermore, the hypotensive group at presentation required more vasopressors than those who presented with systolic $\mathrm{BP} \geq 90 \mathrm{mmHg}$.

There was no difference in SIRS criteria between the hypotensive and normotensive group as $69 \%$ of patients in both groups had greater than 2 SIRS criteria.

\section{Disposition}

Admitted to the ICU were $45.3 \%$ of the patients, and the remaining $54.7 \%$ were managed on the general practice unit (GPU) (Table 4). The mean length of stay in the ED was $13.35 \pm 17.15 \mathrm{~h}$, while it was $12.04 \pm 13.95$ and $7.27 \pm 5.94$ days in the ICU and GPU, respectively. The mean length of stay in the hospital was $13.86 \pm$ 13.62 days (Table 3); $73.1 \%$ of patients with initial systolic blood pressure less than $90 \mathrm{mmHg}$ were admitted to the ICU.

Table 3 Patient management characteristics

\begin{tabular}{|c|c|c|c|}
\hline & Number & Mean \pm SD & Range \\
\hline LOS in ED (hours) & 97 & $13.35 \pm 17.154$ & $1.00-137.50$ \\
\hline LOS in ICU (days) & 43 & $12.04 \pm 13.951$ & $0.40-70.67$ \\
\hline LOS in GPU (days) & 52 & $7.27 \pm 5.941$ & $0.33-30.95$ \\
\hline LOS in the hospital (days) & 97 & $13.86 \pm 13.618$ & $0.507-70.917$ \\
\hline Time to vasopressors in the first $24 \mathrm{~h}$ (hours) & 21 & $9.14 \pm 8.027$ & $0.50-24.00$ \\
\hline Time to norepinephrine (hours) ${ }^{a}$ & 36 & $148.20 \pm 282.190$ & $1-1464.00$ \\
\hline Time to dopamine (hours) ${ }^{a}$ & 7 & $278.31 \pm 393.557$ & $1-870.50$ \\
\hline Time to dobutamine (hours) & 4 & $59.50 \pm 41.026$ & $22-114.50$ \\
\hline Vasopressors Tx duration (days) for those who took vasopressors in the first $24 \mathrm{~h}$ & 21 & $3.33 \pm 3.411$ & $0.13-12.88$ \\
\hline Steroid Tx duration (days) & 47 & $7.55 \pm 7.540$ & $0.50-35.00$ \\
\hline Time to antibiotic treatment initiation (hours) ${ }^{a}$ & 96 & $3.43 \pm 4.479$ & $0.167-36.33$ \\
\hline IV fluid requirement first $6 \mathrm{~h}$ (liters) & 96 & $1.75 \pm 1.964$ & $0.02-11.06$ \\
\hline IV fluid requirement first $24 \mathrm{~h}$ (liters) & 96 & $3.37 \pm 2.846$ & $0.160-16.642$ \\
\hline
\end{tabular}

The time to norepinephrine, to dopamine, and to antibiotic treatment for one patient is missing since the paper containing this information was not found in the patient's chart 
Table 4 Disposition of septic patients

\begin{tabular}{lll}
\hline & Number & Percent \\
\hline Admission $^{\text {a }}$ & 43 & \\
ICU & 52 & 45.3 \\
GPU & 30 & 54.7 \\
Hospital mortality & 67 & 30.9 \\
Discharge home & & 69.1 \\
28-day mortality & 20 & \\
Yes & 19 & 20.6 \\
Unknown & 19.6 \\
\hline
\end{tabular}

${ }^{\mathrm{a}}$ Two patients have not been admitted as one of them left AMA and the other one died in the ED

\section{Mortality}

A total of 30 (30.9\%) septic patients died. The 28-day mortality was $20.6 \%$ with $19.6 \%$ lost to follow-up. There was no statistically significant difference in hospital mortality between the hypotensive and normotensive groups. There was no significant difference in mean age, gender distribution, and comorbidities between the discharged and deceased group. The percentage of bacteremia was significantly higher in the survival group $(92.7 \%)$ than in the deceased group (7.3 \%).

Deceased patients had greater vasopressor use and a longer stay in the ICU $(p=0.001)$. Time to norepinephrine was significantly longer in the deceased group $(p=0.004)$ (Table 5).

Vital signs upon presentation and after $6 \mathrm{~h}$, IV fluids requirements during the first 6 and $24 \mathrm{~h}$, and time to initiation of antibiotics were not different between the two groups. Out of 30 patients who presented initially with less than 2 SIRS criteria, 8 died during their

Table 5 Patient mortality characteristics

\begin{tabular}{|c|c|c|c|c|c|}
\hline & \multicolumn{2}{|c|}{ Mortality (no) } & \multicolumn{2}{|c|}{ Mortality (yes) } & \multirow{2}{*}{$p$ value } \\
\hline & $N$ & $\%$ & $N$ & $\%$ & \\
\hline Norepinephrine & 17 & 45.9 & 20 & 54.1 & $<0.001$ \\
\hline Dopamine & 3 & 37.5 & 5 & 62.5 & 0.044 \\
\hline Steroid use & 25 & 53.2 & 22 & 46.8 & 0.001 \\
\hline Antibiotics initiated in ED & 61 & 71.8 & 24 & 28.2 & 0.127 \\
\hline Antibiotics initiated in ICU & 2 & 33.3 & 4 & 66.7 & 0.248 \\
\hline Antibiotics initiated in GPU & 4 & 53.2 & 2 & 46.8 & 0.248 \\
\hline \multicolumn{6}{|l|}{ SIRS } \\
\hline 0 or 1 & 22 & 73.3 & 8 & 26.7 & \multirow[t]{2}{*}{0.543} \\
\hline$\geq 2$ & 45 & 67.2 & 22 & 32.8 & \\
\hline \multicolumn{6}{|l|}{ Bacteremia } \\
\hline No & 29 & 51.8 & 27 & 48.2 & \multirow[t]{2}{*}{$<0.001$} \\
\hline Yes & 38 & 92.7 & 3 & 7.3 & \\
\hline ICU admission & 24 & 55.8 & 19 & 44.2 & 0.012 \\
\hline GPU admission & 42 & 80.8 & 10 & 19.2 & 0.007 \\
\hline
\end{tabular}

hospital stay (26.7 \%) (Table 5). Laboratory findings also were not significantly different between the two groups.

\section{Discussion}

Since EGDT was published, there has been a steady decline in sepsis-related mortality. The rate of hospitalizations however has doubled during the past decade in the USA [7]. Although much of the therapy for severe sepsis occurs in ICU, as many as 500,000 cases of severe sepsis are initially managed in EDs annually, with an average ED length of stay of $5 \mathrm{~h}[5,6,8]$. Recent research has shown that the most important and the cornerstone of sepsis therapy is early recognition, aggressive fluid hydration and early antibiotics [3, 4]. The surviving sepsis campaign recommends a $30 \mathrm{cc} / \mathrm{kg}$ bolus in the first $3 \mathrm{~h}$ of resuscitation [2]. Further fluid therapy is guided by monitoring various hemodynamic metrics such as the central venous pressure or the mean arterial pressure. The mean amount of fluids given in our cohort within the first $6 \mathrm{~h}$ was $1.75 \mathrm{l}$. There was however a statistically significant difference in fluid resuscitation between the hypotensive and normotensive group. This discrepancy is probably stemming from the prejudice that hypotensive patients are sicker. However, EGDT and SSC recommend aggressive hydration to both severe sepsis and septic shock regardless of blood pressure on presentation. Early antibiotics have been shown to improve survival in septic patients [9]. The mean time to antibiotic in our study was $3.43 \mathrm{~h}$ with the majority $(87.6 \%)$ of antibiotics started in the emergency department. It is important to note that at the time the study was conducted, our institution did not follow yet a standardized protocol-based approach to sepsis management, which would explain why most sepsis resuscitation goals were not met.

According to the literature, the most common infection sites in septic patients are the respiratory and genitourinary systems as well intra-abdominal surgical infections and indwelling catheters [5]. The most common focus of infection in our population was the genitourinary system with Gram-negative organisms being the most predominant culprits. This is in accordance with a previous study done in our institution looking at bacteremia in febrile neutropenia patients that showed the growing incidence of Gram-negative organisms [10].

Septic shock is one of the leaders in mortality nowadays, but with early recognition and treatment, we have seen an improvement in septic shock mortality [2]. The mortality from septic shock was found to be as high as $46 \%$ in the original EGDT control group, but the implementation of protocols aiming at early identification and aggressive care has lead to an improved survival, and nowadays the mortality from septic shock ranges between 20 and $30 \%[1,2,11]$. To the best of our knowledge, there is one study that looked at sepsis and its toll 
in the Middle East. Memon et al. looked at the impact of a 6-h resuscitation bundle on sepsis outcome in a Saudi Arabian intensive care unit and found a reduction in mortality in septic patients from 31 to $21 \%$ [12]. In our cohort, the hospital mortality was $30.9 \%$ and the 28 -day mortality was $20.6 \%$. It was interesting to note that there was no significant mortality difference between the normotensive and hypotensive patients at presentation.

There are a limited amount of ICU beds at our institution. Very often, patients requiring ICU stay tend to remain in the ED for a prolonged time until a bed is available. Should these patients improve during their ED stay, they are downgraded to a GPU bed. This in part explains our long ED length of stay and our high GPU admission rate. Our GPU beds are not equipped with continuous monitoring. Vital signs are taken every 4$6 \mathrm{~h}$. Vasopressors, antibiotics, and non-invasive ventilation can be initiated on the GPU. Further invasive hemodynamic monitoring (CVP, arterial lines) occurs in the critical care units. Of all the patients who were admitted to the GPU, 10 passed away. They were stepped up to an ICU bed and eight of them were successfully moved to the MICU. The two remaining patients passed away on the wards after their families requested to make them comfort care. Overall, patients admitted to the ICU had a greater mortality, and this can be explained by the fact that sicker patients are usually admitted to critical care units.

The SIRS criteria were initially proposed as a screening method to rapidly flag possible septic patients, as sepsis is defined as having 2 or more SIRS criteria in the setting of a presumed or documented infection. Even though they were sensitive, SIRS criteria were not specific and did not correlate with mortality [13]. Owing to the fact that our study was a retrospective one, we selected patients based on their discharge diagnosis and wanted to look at the value of the SIRS criteria at presentation. It is interesting that in our study, $27 \%$ of patients who presented with less than 2 SIRS criteria died within their hospital stay. Emergency physicians should be aware of this and should maintain a high level of suspicion when caring for septic patients presenting with normal vital signs.

\section{Limitations}

This is a retrospective study done in a single emergency department, and as such, our study has many limitations. Some of the patients did not have repeat vitals at $6 \mathrm{~h}$, and lactate was not drawn on all patients as our institution began measuring lactate on septic patients fairly recently. Our sample size is small and there was a significant amount of patients who were lost to followup. Furthermore, information regarding initial ED disposition or whether patients were stepped up to an ICU level bed was not available. This can be explained by our institution's use of paper-based medical records at the time of the study, which might have lead to the loss of some patient information.

\section{Conclusions}

This is the first study looking at an in-depth analysis of sepsis in the Middle Eastern population and examining the overall management of septic patients and their inhospital mortality in a single tertiary care center in Beirut, Lebanon, prior to the initiation of a protocolbased approach. Although our mortality rate was in the range of the Western world, it will be very interesting to see the effect of sepsis treatment bundles advocating for early recognition, aggressive hydration, and early antibiotics on sepsis-related mortality.

We hope that it will stimulate further prospective studies on sepsis in the Middle East and other developing countries with limited healthcare resources.

\section{Competing interests}

The authors declare that they have no competing interests.

\section{Authors' contributions}

GAD and RBC have made substantial contributions to the conception and design of the study. RB, MS, and DZ have made substantial contributions to the acquisition of data, analysis, and interpretation of data. GAD, RBC, and MS have been involved in drafting the manuscript. GAD and RBC revised the manuscript critically for important intellectual content. GAD and RBC have given final approval of the version to be published. All authors read and approved the final manuscript.

\section{Acknowledgements}

The authors have no source of funding to declare.

Received: 18 February 2015 Accepted: 28 May 2015

Published online: 06 June 2015

\section{References}

1. Rivers E, Nguyen B, Havstad S, Ressler J, Muzzin A, Knoblich B, et al. Early goal-directed therapy in the treatment of severe sepsis and septic shock. N Engl J Med. 2001;345(19):1368-77.

2. Levy MM, Dellinger RP, Townsend SR, Linde-Zwirble WT, Marshall JC, Bion J, et al. The Surviving Sepsis Campaign: results of an international guideline-based performance improvement program targeting severe sepsis. Crit Care Med. 2010;38(2):367-74.

3. Pro Cl, Yealy DM, Kellum JA, Huang DT, Barnato AE, Weissfeld LA, et al. A randomized trial of protocol-based care for early septic shock. N Engl J Med. 2014;370(18):1683-93.

4. The Al. the ACTG. Goal-directed resuscitation for patients with early septic shock. N Engl J Med. 2014;371(16):1496-506.

5. Angus DC, Linde-Zwirble WT, Lidicker J, Clermont G, Carcillo J, Pinsky MR. Epidemiology of severe sepsis in the United States: analysis of incidence, outcome, and associated costs of care. Crit Care Med. 2001;29(7):1303-10.

6. Wang HE, Shapiro NI, Angus DC, Yealy DM. National estimates of severe sepsis in United States emergency departments. Crit Care Med. 2007;35(8):1928-36.

7. Gaieski DF, Edwards JM, Kallan MJ, Carr BG. Benchmarking the incidence and mortality of severe sepsis in the United States. Crit Care Med. 2013;41(5):1167-74.

8. Puskarich MA, Marchick MR, Kline JA, Steuerwald MT, Jones AE. One year mortality of patients treated with an emergency department based early goal directed therapy protocol for severe sepsis and septic shock: a before and after study. Crit Care. 2009;13(5):R167. 
9. Gaieski DF, Mikkelsen ME, Band RA, Pines JM, Massone R, Furia FF, et al. Impact of time to antibiotics on survival in patients with severe sepsis or septic shock in whom early goal-directed therapy was initiated in the emergency department. Crit Care Med. 2010;38(4):1045-53.

10. Kanafani ZA, Dakdouki GK, El-Chammas Kl, Eid S, Araj GF, Kanj SS.

Bloodstream infections in febrile neutropenic patients at a tertiary care center in Lebanon: a view of the past decade. Int J Infect Dis. 2007;11(5):450-3.

11. Kaukonen KM, Bailey M, Suzuki S, Pilcher D, Bellomo R. Mortality related to severe sepsis and septic shock among critically ill patients in Australia and New Zealand, 2000-2012. JAMA. 2014;311(13):1308-16.

12. Memon Jl, Rehmani RS, Alaithan AM, El Gammal A, Lone TM, Ghorab K, et al. Impact of 6-hour sepsis resuscitation bundle compliance on hospital mortality in a Saudi hospital. Crit Care Res Pract. 2012;2012:273268.

13. Jaimes F, Garces J, Cuervo J, Ramirez F, Ramirez J, Vargas A, et al. The systemic inflammatory response syndrome (SIRS) to identify infected patients in the emergency room. Intensive Care Med. 2003;29(8):1368-71.

\section{Submit your manuscript to a SpringerOpen ${ }^{\circ}$} journal and benefit from:

- Convenient online submission

$\checkmark$ Rigorous peer review

- Immediate publication on acceptance

- Open access: articles freely available online

- High visibility within the field

- Retaining the copyright to your article

Submit your next manuscript at $>$ springeropen.com 